\title{
Benefits of a coordinated Fog-to-Cloud resources management strategy on a Smart City Scenario
}

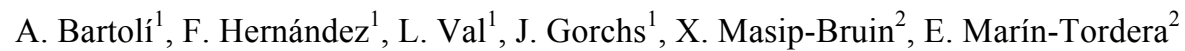 \\ J. Garcia ${ }^{2}$, A. Juan ${ }^{3}$, A. Jukan ${ }^{4}$ \\ ${ }^{1}$ Worldsensing Group, LONDON 9-10 Carlos Place, Mayfair London W1K 3AT, United Kingdom \\ ${ }^{2}$ Advanced Network Achitectures Lab (CRAAX), UPC, Neapolis Buil., Vilanova i la Geltrú, Spain \\ ${ }^{3}$ ATOS Research \& Innovation, Pere IV, Barcelona, Spain \\ ${ }^{4}$ Technische Universität of Braunschweig, Hans-Sommer St, 66, Braunschweig, Germany \\ \{abartoli, fhernandez, lval, jgorchs\} @worldsensing.com \\ \{xmasip,eva,jordig\}@ac.upc.edu; ana.juanf@atos.net; a.jukan@tu-bs.de
}

\begin{abstract}
The advent of fog computing devices as computing paradigm enriching traditional cloud computing applications, paves the way to deploy innovative services, typically not completely appropriate and well supported by cloud computing technology. For example, fog computing is highly suitable for services requiring high constraints on delay, such as dependable services in the e-health arena or tracking strategies in manufacturing processes. Recently, some initiatives have focussed on putting together fog and cloud computing to make the best out of utilizing both, such as the reference architecture by the OpenFog consortium or the Fog-to-Cloud (F2C) concept. However, such a scenario requires a novel management strategy taking over the foreseen specific demands. In this paper, we argue the benefits of a F2C architecture on a particular application to be deployed on a smart city or smart environment scenario.
\end{abstract}

Keywords. Cloud computing, fog computing, F2C computing, coordinated management.

\section{Introduction}

Several efforts have been done recently analyzing the complex scenario brought by putting together cloud and fog resources, such as Fog-to-Cloud (F2C) [1] or the recent OpenFog reference architecture [2], growing from a logical evolution in the cloud arena as shown in Fig. 1.

The main rationale for this scenario boils down to using resources best suiting expected services demands, be it at the cloud or at the fog, to support services execution and related quality (QoS). Indeed, the advent of fog computing [3] has paved the way to introduce novel concepts that are undoubtedly impacting on services performance in typical smart environment. Particularly relevant are the effects on reducing latency, improving security 
and limiting the traffic to be sent throughout the core network to reach out the cloud. When fog and cloud are put together, a new scenario is envisioned, setting a stack of resources

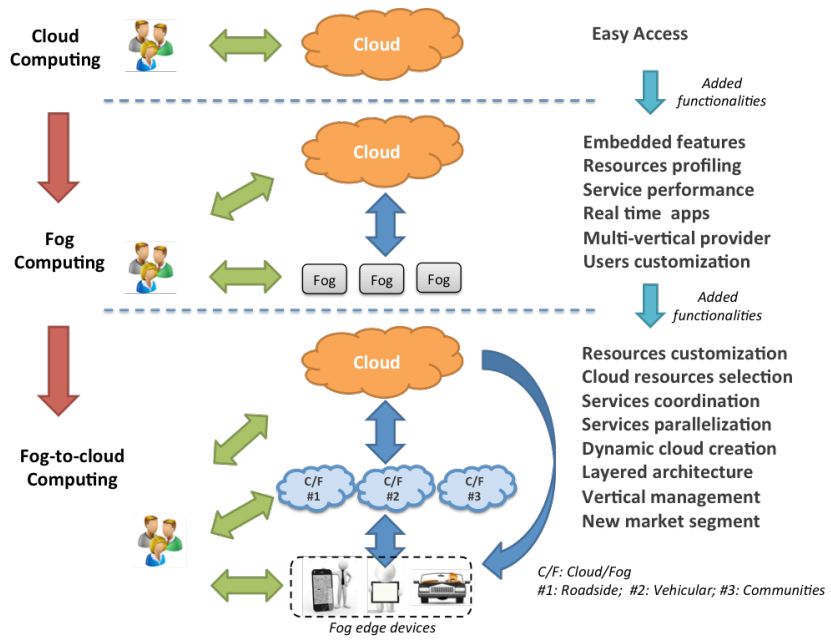

Fig. 1. Cloud evolution towards fog-to-cloud computing (from [1])

(Fig. 2), where the resources must be managed in a coordinated fashion to facilitate an optimal match between technology capacities and services needs/demands

Moreover, such a coordinated management layer may also set the roots for new services execution strategies based on sub-services decomposition, aggregation and parallelization techniques. The effects of such an innovative strategy will have a large impact on the development of new business models, novel market opportunities and unquestionably new avenues for research; for example, devices naming, services allocation or resources categorization, etc. It is worth noticing that the fog scenario is strongly aligned to consider edge devices, what usually strongly links to mobility aspects. Mobility indeed brings undesired constraints on resources management, such as volatility and availability, what when added to the devices heterogeneity sets a very complex scenario.

In this context, the $\mathrm{mF} 2 \mathrm{C}$ project [4], which is an EU Research and Innovation action

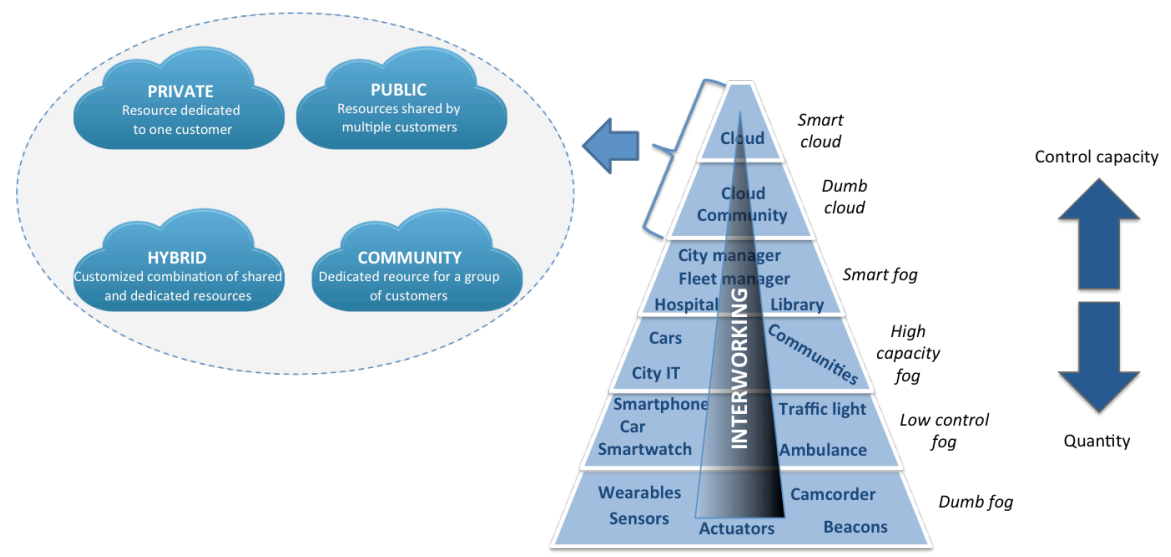

Fig. 2. The stack of resources architecture envisioned in F2C 
funded by the Horizon 2020 program, aims to design, implement and validate novel management architecture and methodology for achieving the $\mathrm{F} 2 \mathrm{C}$ paradigm. Within this initiative, the demonstration part is supported by the deployment of the $\mathrm{mF} 2 \mathrm{C}$ project's outcomes into three different use cases; each use case will bring a different set of characteristics where the $\mathrm{mF} 2 \mathrm{C}$ paradigm is expected to show its main capabilities and benefits. This paper is intended to overview the main characteristics for one of the proposed $\mathrm{mF} 2 \mathrm{C}$ use cases as well as to envision the benefits brought by considering a successful deployment of the $\mathrm{mF} 2 \mathrm{C}$ management solution within the selected experimentations. In a nutshell, the proposed use case focuses on a scenario of emergency management enriched with $\mathrm{mF} 2 \mathrm{C}$ innovation to provide added value for the smart city ecosystem: customers, companies, governments, citizens, etc.

The paper is structured as follows. Section 2, briefly introduces the $\mathrm{mF} 2 \mathrm{C}$ architecture. Section 3 draws the proposed scenario and Section 4 identifies main expected benefits. Finally, Section 5 concludes the paper.

\section{Preliminary $\mathbf{m F 2 C}$ architecture}

In general, the main deployment for the $\mathrm{mF} 2 \mathrm{C}$ vision and architecture will turn into management agents, which will be deployed on a device to be $\mathrm{mF} 2 \mathrm{C}$-capable. The strategy to define how devices joint $\mathrm{mF} 2 \mathrm{C}$, are discovered and identified, clustered and categorized, etc. is still under research. However, a preliminary version of the main building blocks of the $\mathrm{mF} 2 \mathrm{C}$ architecture is shown in Fig. 3. The tentative architecture considers three main blocks which work together to allocate the different expected control and management functionalities. Notably, the main blocks are called:

- Controller: Includes the set of control and management functions, distributed in various architectural entities.

- Gearbox: Includes the set of policies, strategies, configurations, etc., enriching $\mathrm{mF} 2 \mathrm{C}$, and paving the way to adopting novel strategies, such as service orchestration, runtime systems, etc.

- Interfaces: Different interfaces are envisioned to guarantee internal and external openness.

More in detail, the proposed set of control and management blocks include:

\subsection{Controller Block}

This block splits into three main components, resources, services and user, putting all together the set of expected functionalities to deploy the whole Controller Block. They include aspects yet under active research, such as semantic adaptation, resource management (monitoring discovering, virtualization, etc.), security and privacy, etc. It is worth noticing that the dynamicity inherent to $\mathrm{F} 2 \mathrm{C}$ resources, the heterogeneity foresaw for the devices and systems comprising $\mathrm{mF} 2 \mathrm{C}$ as well as the business relationship to be established among resources providers makes these aspects very challenging. Moreover, the matching between resources available and services demands is also posing several challenges; for example, in resources/services taxonomy, categorization, mapping and final allocation, considering active policies on resources provisioning while always providing the expected quality (QoS) as defined in the Service Level Agreement (SLA) set with the final 
user to run the expected service. The proposed taxonomy should be dynamic enough to accommodate future developments. When required, the service can be decomposed (atomized) into sub-services, turning into a set of atomic services (sub-services), commonly requiring fewer resources and facilitating new approaches, such as parallel execution. The set of sub-services may be preconfigured and stored in a repository. Challenging issues in this area include: i) to find the appropriate place to locate the service decomposition, ii) to minimize the computing load while keeping fast reaction time, iii) to define what extent these functions must be associated to the aggregation points, iv) to define the graph rules including not only the sub-services but also their dependencies and strategies for subservices search, etc.

Finally, considering security, mF2C must benefit from the user-specific context information to tailor service execution to the specific user demands. To that end, a comprehensive set of functionalities must be defined, including but not limited to authentication, privacy, location, profiling, agreement policies, etc. (i.e., User and Context Functions). All these functionalities must meet the business policies in place to guarantee real $\mathrm{mF} 2 \mathrm{C}$ deployment.

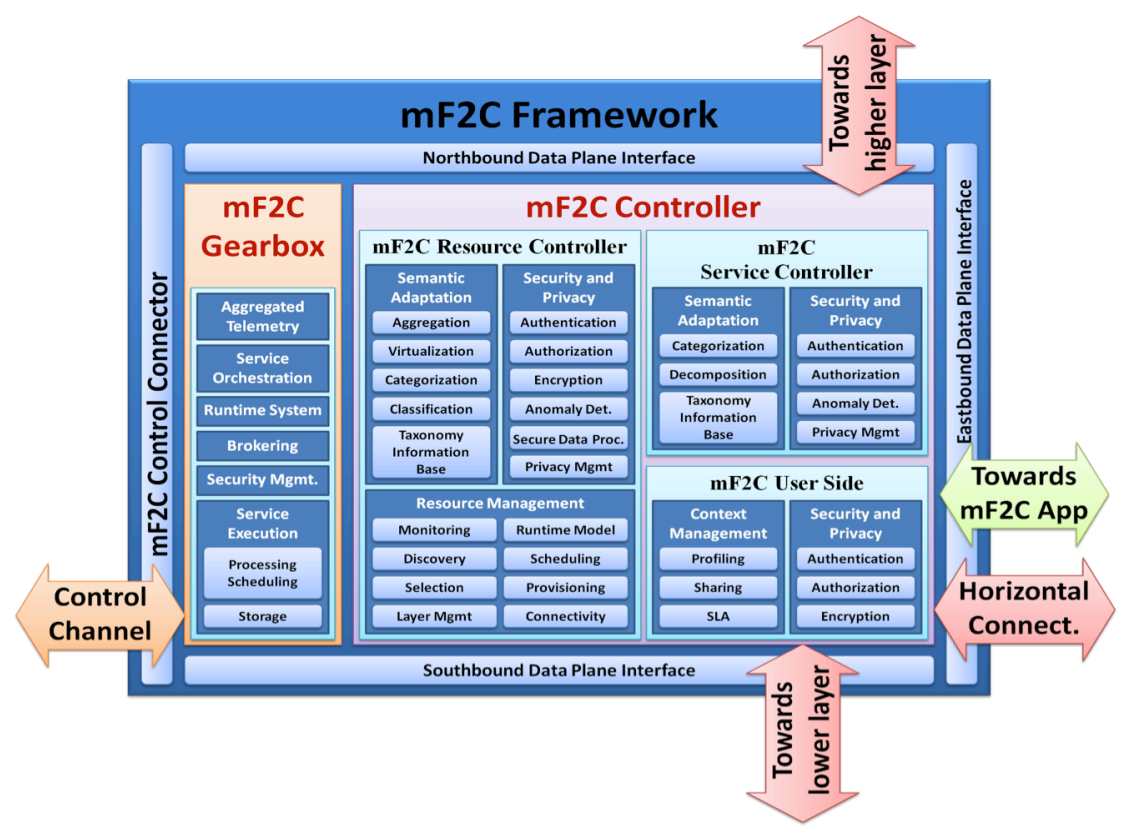

Fig. 3. Architectural blocks for the $\mathrm{mF} 2 \mathrm{C}$ management framework (from $\mathrm{mF} 2 \mathrm{C}$ project)

\subsection{Gearbox Block}

The set of preliminary components defined to build the Gearbox block focus on two main components: monitoring and service orchestration. The first is required to inform decision making systems such as service orchestration; it should be dynamically configurable, and support derived or aggregated metrics at the edge for maximum scalability of the overall 
solution. The second is responsible for allocating services to the best suitable resources; the optimal allocation will depend on many factors, such as historical analysis, real-time resources configuration, QoS, etc.

\subsection{Interfaces}

Different interfaces are envisioned to ease the $\mathrm{mF} 2 \mathrm{C}$ interconnectivity and interoperation. The whole system must support a northbound and southbound interface to vertically facilitate connection within the F2C architecture. The Eastbound interface enables multicloud/fog communication within the same layer and the westbound interface is envisioned for control functionalities.

\section{The Smart City Scenario}

Worldsensing will take advantage of $\mathrm{mF} 2 \mathrm{C}$ project by implementing secure and powerful specific IoT services based on a prolific relation between end-devices and a central platform. This SME is interested in both the scalable and flexible approach brought by the $\mathrm{mF} 2 \mathrm{C}$ resources management and its suitability to deploy services out of a data-center, and therefore move the computation close to the origin of data. The synergies and the knowledge obtained in the proposed use case will enable more ambitious developments to thus allow the access to wider markets that are not currently explored by Worldsensing due to the limited availability of cost-effective technology in this context. In this section, we will present Worldsensing group and its use case within $\mathrm{mF} 2 \mathrm{C}$.

\section{1 Worldsensing Group}

Within the mF2C project, one of the use cases is brought by Worldsensing group. Worldsensing was founded in 2008 and currently employs more than 60 people in Barcelona and London premises. It enjoys exponentially growing sales worldwide: it concluded its Serie-A investment in early 2013; in 2016, it received a Serie-B inversion from international parties; and, in 2017, it is currently focusing on a novel round of Serie-C investment. The great grow of Worldsensing group is depending on its main expertise: the company provides high-quality sensing and machine-to-machine technologies and services to specific industry verticals. It has two product portfolios: one being Smart Traffic solutions for Smart Cities, and the other being Heavy-Industry Monitoring solutions. As for smart traffic, it counts on its own smart parking product and a journey-time monitoring solution through its acquisition of smart traffic giant Bitcarrier. As for heavy-industry monitoring, it instruments critical infrastructures such as buildings, bridges, tunnels, ports, wells, etc. and offers seismic monitoring capabilities for engineering, oil/gas/water acquisition and $\mathrm{CO} 2$-sequestration purposes.

Worldsensing is currently market leader in most of above M2M/IoT industry monitoring markets. It has won numerous prizes and awards, has enjoyed vast press coverage by the WSJ and the BBC, has shaped many IoT/M2M standards, and has driven the R\&D developments in Europe through the participation of numerous FP7 and H2020 projects. 


\subsection{Emergency Situation Management in a Smart City (ESM)}

Continuing into this century, society has supported a movement of people from rural areas to cities. Nowadays, more people live in urban environments than in rural ones. It is estimated that this process will not stop and within 20 years the urban population will be around 5 billion. The big challenges for the whole society will be related to resource management in these overcrowded environments. Worldsensing works in the development of a model for smart cities and its products intend to solve current and future problems related to the use of innovative technology to challenge emergency management.

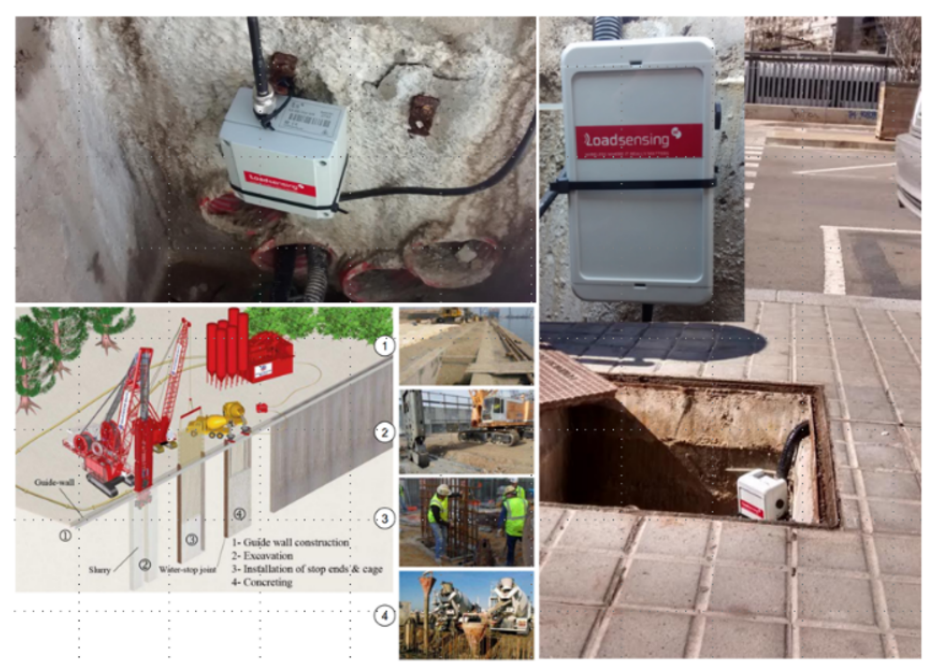

Fig. 4. WOS IoT industrial device

The proposed "Emergency Situation Management" use case within $\mathrm{mF} 2 \mathrm{C}$ project aims to validate a novel and innovative hybrid architecture that serves i) to analyse flows of people and infrastructure state in order to provide useful information to private customers and authorities, ii) to detect a possible emergency in real-time, and iii) to decrease the necessary resources in terms of energy, latency, etc. to respond to specific situations in accordance with the applications requirements.

The proposed use case is based on the implementation of distributed elements of capturing signals and data (Worldsensing IoT device, see Fig. 4) as well as a centralized asset management system to integrate heterogeneous industrial-related information in a flexible and efficient cloud platform (Worldsensing asset management platform, see Fig. 5). The $\mathrm{mF} 2 \mathrm{C}$ paradigm should suggest policies to manage the aforementioned hybrid architecture where services and decisions have to be taken in real-time for improving the performance of today industrial systems. 


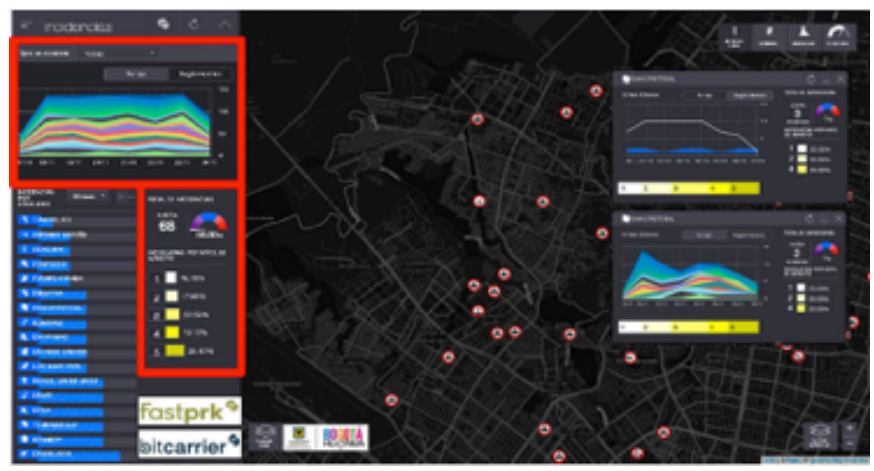

Fig. 5. WOS asset management platform

Normally, the information captured by today IT (Information Technology) and OT (Operational Technology) devices is stored and processed in a cloud computing system to present such information to the end user. Occasionally, in case of emergency situations, the information captured by the devices is processed through FOG devices to optimise the Quality of Service (QoS) factors. In mF2C project, a complete approach where asset management and control data are considered in the same solution suite.

The proposed use case is subdivided into 3 phases: i) To assess the performance of a central industrial management system in terms of key parameters in normal operations: latency, reliability, data elaboration, etc. (Cloud Computing assessment); ii) To assess the performance of monitoring devices considering specific requirements when emergency situation are detected, i.e. an accident (Fog computing assessment); and iii) To assess the $\mathrm{mF} 2 \mathrm{C}$ solution, where a hybrid framework guarantees high Fog and Cloud computing performance, both during ordinary operation and emergency management situation. The results have to be at least similar to point 1 and 2 where the centralized and distributed problems are solved in isolated fashion ( $\mathrm{mF} 2 \mathrm{C}$ model assessment).

Finally, Fig. 6 shows the technological perspective deployed in the use case. This last

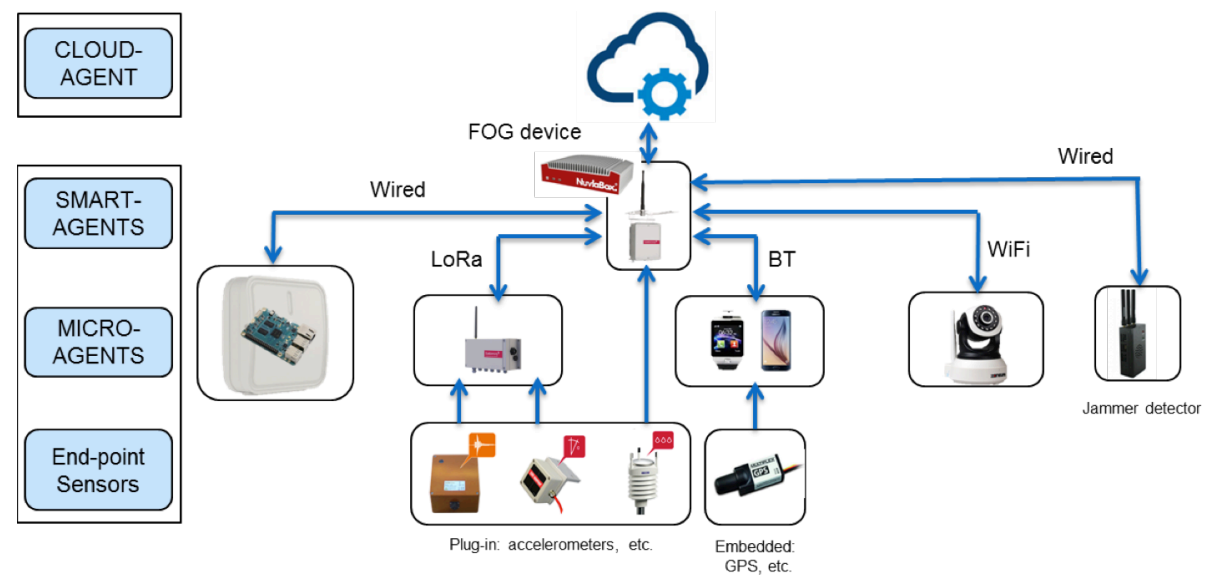

Fig. 6. Technologies deployment in the Smart City scenario 
figure shows the different components provided by Worldsensing and $\mathrm{mF} 2 \mathrm{C}$ consortium as well as its location in the proposed Smart City scenario.

\section{Potential impact brought by mF2C}

The Smart City use case aims to integrate industrial solutions to provide advanced and more efficient services to both end-users and decision makers. The introduction of $\mathrm{mF} 2 \mathrm{C}$ paradigm within the Smart City ecosystem will provide more scalable and secure commercial solutions. Indeed, the $\mathrm{mF} 2 \mathrm{C}$ implementation will enable progressive scaling of the infrastructure complexity and can absorb the increasing needs of computational demand (such as due to big data). This is very relevant, considering market opportunities and business forecast in Smart Cities and IoT. The developed management system will facilitate scaling and lowering infrastructure cost, especially for the envisioned smaller-scale deployments that today are too expensive due to the need for over-dimensioned dedicated infrastructure. The $\mathrm{mF} 2 \mathrm{C}$ solution will also enable the introduction of new computing paradigms, including edge-technology computing approach, where computation is moved close to the "edges" of the Internet to reduce risks of failure and delay, and improve reliability in the sense that data is not lost while the edge device is temporarily disconnected. This approach will enable cities to install fog computing infrastructure locally and enable new real-time services. More concretely the mF2C benefits in the context of managing Emergency Situations in smart cities may be summarized as:

- Increasing service reliability by $30 \%$ and Quality of service (QoS) by $10 \%$

- Decreasing delays by $30 \%$

- Decreasing operational costs (OPEX) by $10 \%$

Worldsensing will take advantage of the $\mathrm{mF} 2 \mathrm{C}$ framework to build secure and powerful specific IoT services based on a prolific relation between end-devices and central platform. The synergies and the funding obtained in the project will enable more ambitious developments which will permit to access to wider markets that are not touched currently due to the limited availability of suitable platforms. The issues addressed in this project will be of great importance to improve energy-efficiency, robustness, ease-of-use and security required for meeting the needs of the Worldsensing's roadmap towards deploying applications in urban and industrial scenarios. In particular, $\mathrm{mF} 2 \mathrm{C}$ achievements will allow us to design new IoT services, therefore, creating new and strengthening considerably existing business lines.

\section{Conclusions}

In this paper, we describe the main impact brought by deploying a coordinated management strategy for a scenario combining fog and cloud resources, on a specific service addressing the management of emergency situations in a smart city. The presented benefits do not only focus on the specific service itself, but also put the focus on the impact it may have on a well-established company deploying such a service. Thus, the main objective of the paper does not deal with conceptually deploying architecture to support 
such a management, what is briefly introduced in the paper, but on highlighting the benefits brought by such a deployment. Certainly, the proposed scenario is just one vertical showing the foreseen benefits, and many others are also envisioned, such as on the e-health area or in smart transportation.

\section{Acknowledgements}

This work is supported by the H2020 mF2C project (730929). For UPC authors is also supported by the Spanish Ministry of Economy and Competitiveness and by the European Regional Development Fund under contract TEC2015-66220-R (MINECO/FEDER).

\section{References}

1. X.Masip, E.Marín, A.Jukan, G.J.Ren, G.Tashakor, "Foggy clouds and cloudy fogs: a real need for coordinated management of fog-to-cloud (F2C) computing systems", IEEE Wireless Communications Magazine, October 2016

2. Openfog reference architecture at https://www.openfogconsortium.org/ra/

3. F. Bonomi, R. Milito, P. Natarajan, J. Zhu, "Fog Computing: A Platform for Internet of Things and Analytics", N. Bessis and C. Dobre (eds.), Big Data and Internet of Things: A Roadmap for Smart Environments, Studies in Computational Intelligence 546, DOI: 10.1007/978-3-31905029-4 7, Springer International Publishing Switzerland 2014

4. $\mathrm{mF} 2 \mathrm{C}$ project at http://www.mf2c-project.eu 